\title{
Two-Stage Trajectory Optimization for Autonomous Ground Vehicles Parking Maneuver
}

\author{
Runqi Chai, Member, IEEE, Antonios Tsourdos, Al Savvaris, Senchun Chai, and Yuanqing Xia
}

\begin{abstract}
This paper proposes a two-stage optimization framework for generating the optimal parking motion trajectory of autonomous ground vehicles. The motivation for the use of this multi-layer optimization strategy relies on its enhanced convergence ability and computational efficiency in terms of finding optimal solutions under the constrained environment. In the first optimization stage, the designed optimizer applies an improved particle swarm optimization technique to produce a near-optimal parking movement. Subsequently, the motion trajectory obtained from the first stage is used to start the second optimization stage, where gradient-based techniques are applied. The established methodology is tested to explore the optimal parking maneuver for a car-like autonomous vehicle with the consideration of irregularly parked obstacles. Simulation results were produced and comparative studies were conducted for different mission cases. The obtained results not only confirm the effectiveness but also reveal the enhanced performance of the proposed optimization framework.
\end{abstract}

Index Terms-Two-stage optimization, optimal parking trajectory, autonomous ground vehicles, particle swarm optimization, irregularly parked obstacles.

\section{INTRODUCTION}

$\mathbf{M}$ OTION planing or trajectory design problems have been widely researched over the last ten years due to their increasingly significance in industry and military fields [1]-[4]. A high-quality/well-designed trajectory is usually a key for stable movement and design of advanced control systems [5], [6]. Relative works on this topic can be found in a number of engineering practices, such as mobile robot movement design [7], [8], space vehicle maneuver planning [9]-[11], and autonomous ground vehicle dynamic missions [12], [13]. More precisely, the authors in [7] constructed a Riemannian metric-based approach to plan the path for trackedrobot on the raw point cloud. In addition, a collision-free space maneuver robot motion planning problem was established and addressed in [9], wherein a hybrid path planning strategy incorporating a collision detection algorithm and a polynomial interpolation technique was applied to produce feasible motion trajectories. Besides, Yin and Chen [12] studied an autonomous wheeled vehicle motion planning task by applying the spatio-temporal template.

R. Chai, A. Tsourdos and A. Savvaris are with the School of Aerospace, Transport and Manufacturing, Cranfield University, UK, e-mail: (r.chai@cranfield.ac.uk), (a.tsourdos@cranfield.ac.uk), and (a.savvaris@cranfield.ac.uk).

S. Chai and Y. Xia are with the school of Automation, Beijing Institute of Technology, Beijing, China, e-mail: (chaisc97@163.com), (xia_yuanqing@bit.edu.cn).
The mission solved in the present work is a minimumtime parking trajectory planning for a wheeled vehicle. The core aim of this problem is to generate a path/trajectory, for the given autonomous vehicle, to reach the specified parking area in the shortest time without colliding other vehicles/obstacles in the environment. Although extensive research work has been carried out on the design of trajectories for different mission profiles and many effective methods were successfully developed for producing the path, it is only in the recent five years that there has been a growing interest in planning trajectories via optimization-based strategies [14], [15]. The motivation for the use of this kind of technique is that in many real-world trajectory design scenarios, not only the path feasibility should be preserved but also various performance indices are desired to be optimized. For this reason, in this paper, special attentions are given to the implementation of optimization-based trajectory design methods.

It should be noted that currently, there are mainly two types of optimization algorithms: the intelligent methods and traditional gradient-based methods. Contributions made to implement different optimization techniques in autonomous vehicle path generation problems can be found in the literature [16]-[20]. For example, Roberge et al. [16] combined genetic algorithm with particle swarm optimization (PSO) so as to generate near-optimal trajectories for fixed-wing unmanned aerial vehicles in 3-D environment. Similarly, Kim and Lee [17] optimized the manipulator motion by using a PSO algorithm with modified initialization strategy. However, a main disadvantage of applying these intelligent optimization algorithms is that the computation effort required for the optimization process is usually high and can hardly be afforded online.

On the other hand, $\mathrm{Li}$ et al. [18] generated the optimal motions of a wheeled vehicle based on traditional interior point method (IPM) for fulfilling an automatic parking task. A similar application of IPM can also be found in [19], where a higher-order manipulation optimal motion planning problem was solved successfully. Andreas et al. [20] formulated a multi-vehicle energy-optimal motion optimization problem with the consideration of obstacle avoidance and solved it by implementing a barrier functional-based gradient algorithm. Although these results confirmed the effectiveness of using gradient-based optimization techniques, the sensitivity associated with the initial guess value is high and tends to increase the computational time significantly.

To effectively deal with the issue of using intelligent or traditional gradient-based optimization algorithms, a multistage optimization framework is designed to optimize the 
parking movement in this study. The novelty of the proposed computational framework is to incorporate an initial trajectory generator with the gradient-based inner optimizer. The technique used for this reference trajectory generator is a modified PSO method with enhanced local exploitation ability. Compared with traditional single-stage optimization strategy, using the proposed structure tends to have improved convergence performance and reduced computational cost. This will be shown in the simulation section of this paper.

The rest of this paper will be organized as follow: Sec II constructs the mathematical formulation of the time-optimal wheeled vehicle automatic parking problem as well as the collision-free constraints. Following that, in Sec III, the twostage computational optimization framework is introduced. Detailed simulation results and comparative studies are illustrated in Sec IV. Finally, the concluding remark is given in Sec V.

\section{Autonomous Ground Vehicle PARKing OPTIMIZATION PROBLEM}

In this section, the parking optimization model of the wheeled vehicle is formulated. Priory to presenting in detail the formulation of the parking optimization problem studied in this research, it is also worth recalling some related works regarding different vehicle models and mission scenarios investigated in the literature. Currently, there exist various vehicle dynamic systems that can be applied to describe or control the movement of intelligent vehicles. For instance, in [21], a threedegree-of-freedom vehicle dynamic model was constructed. This model was then applied in order to develop a mainservo loop integrated chassis control system. Considering the nonholonomic constraint that limits the wheels to roll with no slip, a kinematic car-like model was established and used in [22] to plan the trajectory for the autonomous lane change maneuver. In addition, an integrated vehicle dynamic model containing the visual recognition system, electrical servo braking system, and steering system was constructed in [23]. Based on this integrated model, a nonsingular fast terminal sliding mode-based emergency braking control strategy was designed. For the parking trajectory planning problem considered in this paper, alternatively, we use kinematics of a car-like vehicle to plan the time-optimal parking trajectories.

\section{A. Vehicle Kinematic Model}

In order to describe the movement of a front-steering vehicle, the equations of motion are firstly constructed. The vehicle is considered as a rigid-body and the sideslip problem is ignored. As a result, its equations of motion can be described as the following system of differential equations:

$$
\left\{\begin{array}{l}
\dot{p}_{x}(t)=v(t) \cos (\theta(t)) \\
\dot{p}_{y}(t)=v(t) \sin (\theta(t)) \\
\dot{v}(t)=a(t) \\
\dot{a}(t)=\operatorname{jerk}(t) \\
\dot{\theta}(t)=v(t) \tan (\phi(t)) \frac{1}{l} \\
\dot{\phi}(t)=\omega(t)
\end{array}\right.
$$

In Eq.(1), $\left(p_{x}, p_{y}\right)$ is the central point of the rear wheel. $t \in\left[0, t_{f}\right]$ denotes the time; $v$ and $a$ are the vehicle velocity and acceleration variables, respectively. $\theta$ stands for the oriental angle, whereas $\phi$ refers to the steering angle with regard to the steering wheel. For brevity reasons, the state variables are defined as $x=\left[p_{x}, p_{y}, v, a, \theta, \phi\right]^{T} \in \mathbb{R}^{6}$. The control variables are composed by the jerk and the front wheel angular velocity $\omega$. That is, $u=[\text { jerk, } \omega]^{T} \in \mathbb{R}^{2}$. To better illustrate the vehicle reference frame, a detailed illustration is plotted in Fig.1. Other vehicle-dependent parameters appeared in Fig.1 are the front overhang length $n$, length between the front and rear wheel $l$, rear overhang $m$, and the vehicle width $2 b$, respectively.

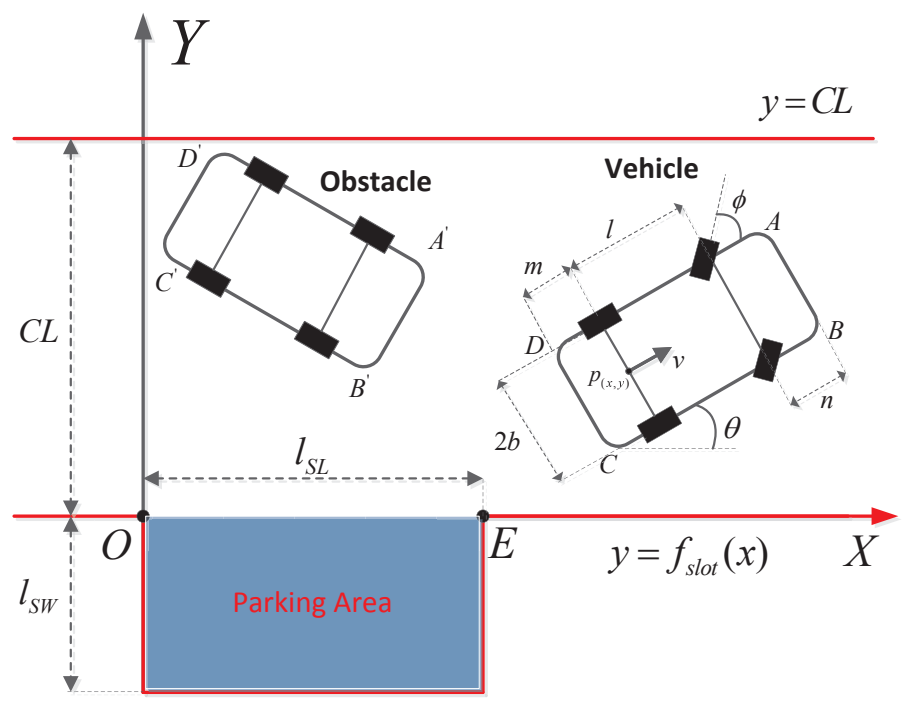

Fig. 1: Automatic parking mission

\section{B. Automatic Parking Process Constraints}

Several mission constraints should be satisfied during the vehicle movement.

1). State constraints: The tolerable range of the state variables can be described as:

$$
\left\{\begin{array} { l } 
{ p _ { x } ( t ) \in [ p _ { x } ^ { \operatorname { m i n } } , p _ { x } ^ { \operatorname { m a x } } ] } \\
{ p _ { y } ( t ) \in [ p _ { y } ^ { \operatorname { m i n } } , p _ { y } ^ { \operatorname { m a x } } ] } \\
{ v ( t ) \in [ v ^ { \text { min } } , v ^ { \text { max } } ] }
\end{array} \quad \left\{\begin{array}{l}
a(t) \in\left[a^{\min }, a^{\max }\right] \\
\theta(t) \in\left[\theta^{\min }, \theta^{\max }\right] \\
\phi(t) \in\left[\phi^{\text {min }}, \phi^{\max }\right]
\end{array}\right.\right.
$$

It should be noted that these state constraints are not just included to make the problem more complex but they do exist in reality. For example, in order to have enough reaction time for potential emergencies, the vehicle should maneuver at a relatively low speed. Hence, a box constraint is assigned to the vehicle speed. Besides, it is not desired to have a significant variance in terms of the speed $v(t)$ as it brings discomfort to the drivers or passengers. Therefore, certain limits should be given to the magnitude of $a(t)$.

2). Control constraints: Certain requirements should also be given to the control variables. For example,

$$
\begin{aligned}
& |\operatorname{jerk}(t)| \leq d_{a}^{\max } \\
& |\dot{k}(t)| \leq d_{k}^{\max }
\end{aligned}
$$

In Eq.(3), $k=\frac{\tan \theta}{l}$ stands for the instantaneous curvature, whereas $\dot{k}=\frac{\omega}{l \cos ^{2} \theta}$ is the corresponding derivative. $d_{a}^{\max }$ and $d_{k}^{\max }$ are the maximum allowable values of the jerk 
variable and $\dot{k}$, respectively. The aim for imposing a constraint on the jerk variable is to smoother the actual acceleration profile. As for the second control constraint (3b), similarly, it can avoid non-smooth segment in the trajectory, thereby improving the ride comfort.

3). Parking area constraints and terminal conditions: To place the vehicle in the specific parking area shown in Fig.1, constraints should be imposed during the vehicle maneuver and at the terminal time instant. Since rigid-body is treated as a rectangular in the 2-D plane, the four corners can be expressed by:

$$
\left\{\begin{array}{cc}
\left(A_{x}, A_{y}\right)= & \left(p_{x}+\cos (\theta)(l+n)-b \sin (\theta),\right. \\
& \left.p_{y}+\sin (\theta)(l+n)+b \cos (\theta)\right) \\
\left(B_{x}, B_{y}\right)= & \left(p_{x}+\cos (\theta)(l+n)+b \sin (\theta),\right. \\
& \left.p_{y}+\sin (\theta)(l+n)-b \cos (\theta)\right) \\
\left(C_{x}, C_{y}\right)= & \left(p_{x}-m \cos (\theta)+b \sin (\theta),\right. \\
& \left.p_{y}-m \sin (\theta)-b \cos (\theta)\right) \\
\left(D_{x}, D_{y}\right)= & \left(p_{x}-m \cos (\theta)-b \sin (\theta),\right. \\
& \left.\left.p_{y}-m \sin (\theta)\right)+b \cos (\theta)\right)
\end{array}\right.
$$

After the definition of corner points, if a successful parking maneuver is achieved, the following inequality constraints should hold:

$$
\left\{\begin{array}{l}
f_{\text {slot }}\left(A_{x}\right) \leq A_{y} \leq C_{L} \\
f_{\text {slot }}\left(B_{x}\right) \leq B_{y} \leq C_{L} \\
f_{\text {slot }}\left(C_{x}\right) \leq C_{y} \leq C_{L} \\
f_{\text {slot }}\left(D_{x}\right) \leq D_{y} \leq C_{L}
\end{array}\right.
$$

where $C_{L}$ is the width of the road. $f_{\text {slot }}$ is given by $f_{\text {slot }}(x)=$ $-\left(H(x)+H\left(x-l_{S L}\right)\right) l_{S W}$. Here, $H(x)$ is the unit jump function. $l_{S L}$ and $l_{S W}$ represent the length and width of the parking area. Using the function $f_{\text {slot }}(x)$ can effectively describe the frontier of the desired parking area, and this function can easily be obtained via the linear combination of the translation and reflection of $H(x)$. Similarly, $y=C L$ is applied to describe the frontier on the other side of the road. The inequality (5) indicates the vehicle should move below $y=C L$ but above $y=f_{\text {slot }}(\cdot)$ during the entire time evolution.

To complete the entire mission, some state variables are required to reach specific values at the terminal time instant. That is,

$$
v\left(t_{f}\right)=0, \quad a\left(t_{f}\right)=0
$$

This implies the automatic parking will end with a full stop.

4). Obstacle avoidance constraints: In this research, we are interested in finding optimal parking trajectories in the presence of irregularly placed obstacles (see obstacle $A^{\prime} B^{\prime} C^{\prime} D^{\prime}$ in Fig.1). To avoid colliding with other vehicles, obstacle avoidance constraints should be designed and entailed in optimization formulation. This is achieved by restricting that any corner point of one rectangular should be outside the other rectangular area. It is worth mentioning that if the following inequality holds true, then the edge point $A$ can locate outside the obstacle $A^{\prime} B^{\prime} C^{\prime} D^{\prime}$.

$$
S_{A^{\prime} A B^{\prime}}+S_{B^{\prime} A C^{\prime}}+S_{C^{\prime} A D^{\prime}}+S_{A^{\prime} A D^{\prime}} \geq S_{A^{\prime} B^{\prime} C^{\prime} D^{\prime}}
$$

In Eq.(7), $S$ stands for the area operation. As a result, if we use Eq.(7) to avoid the collision between the vehicle and the obstacle, eight inequalities should be imposed. Furthermore, during the optimization process, two additional collision-free constraints should also be taken into account in case that the vehicle will not hit the edge of the target parking slot (e.g. $O=(0,0)$ and $\left.E=\left(l_{S L}, 0\right)\right)$. That is, the point $O$ and $E$ should locate outside the vehicle rectangular area:

$$
\left\{\begin{array}{l}
S_{A O B}+S_{B O C}+S_{C O D}+S_{A O D} \geq S_{A B C D} \\
S_{A E B}+S_{B E C}+S_{C E D}+S_{A E D} \geq S_{A B C D}
\end{array}\right.
$$

Remark 1. It should be noted that in [15], the authors achieved the collision-free with respect to $O$ and $E$ by transforming these two points to the vehicle's body frame. However, this intuitive restriction of the slot corner might introduce discontinuity, which will have negative effects for the optimization solver. Alternatively, we use an equivalent but continuous version shown in Eq.(8) to describe it in this study.

\section{Objective and Overall Optimization Formulation}

Since it is desirable to fulfill the parking movement in the shortest time, minimizing $t_{f}$ is selected as the main objective function. The objective function $J$, together with the physical path constraints and obstacle avoidance constraints, formulates the automatic parking maneuver optimization model. The overall formulation is summarised as:

$$
\begin{array}{lll}
\text { minimize } & J=t_{f} & \\
\text { subject to } & \forall t \in\left[t_{0}, t_{f}\right] & \\
& \text { Eq.(1) } & \text { (dynamic constraints) } \\
& \text { Eq.(2),Eq.(3) } & \text { (state/control constraints) } \\
& \text { Eq.(5) } & \text { (path constraints) } \\
& \text { Eq.(6) } & \text { (terminal condition) } \\
& \text { Eq.(7),Eq.(8) } & \text { (collision-free constraint) }
\end{array}
$$

\section{TWO-StAgE TRAJECTORY OPTIMIZATION FRAMEWORK}

In this section, the two-stage trajectory design method is introduced and applied to address the autonomous vehicle parking maneuver problem. The discretized version of the optimal parking problem is firstly defined in Sec III.A. Then an initial parking movement generator is designed in Sec III.B so as to produce a feasible and near-optimal parking trajectory. Subsequently, the generated initial parking trajectory is provided to the inner gradient optimization solver which will be discussed in Sec III.C. The overall structure of this two-stage method is summarised in Sec III.D.

\section{A. Discretized Optimal Parking Model}

It is important to remark that the optimal control model (9) is not solvable in its present form. In order to optimize the control variables of the automatic parking problem, a necessary procedure is to parameterize the continuous-time model. Suppose that the time interval $\left[0, t_{f}\right]$ is divided into $N_{k}$ segments. The goal of the optimization becomes finding the optimal control values at all the discrete time instants subject to the terminal time can be minimized and the constraints can be satisfied. The state can be obtained by integrating 
the dynamics numerically. That is, the optimization model becomes [10]:

$$
\begin{array}{cl}
\operatorname{minimize} & J=t_{N_{k}} \\
\text { subject to } & \forall t_{k}, k \in\left\{1,2, \ldots, N_{k}\right\} \\
& x_{k+1}=x_{k}+h_{k} \sum_{i=1}^{s} q_{i} f\left(x_{k i}, u_{k i}\right) \\
& C\left(x_{k}, u_{k}\right) \leq 0 \\
& \Phi\left(x_{N_{k}}, t_{N_{k}}\right)=0 \\
& i=1, \ldots, s
\end{array}
$$

in which $C(\cdot, \cdot)$ stands for the general form of inequality constraints, whereas $\Phi\left(x_{N_{k}}, t_{N_{k}}\right)=\left[v_{N_{k}}, a_{N_{k}}\right]^{T}$ is the terminal condition. $q_{i}$ is the discretization coefficient and $s$ is the stage of the integration. $x_{k j}$ and $u_{k j}$ are the intermediate variables defined on the current time interval $\left[t_{k}, t_{k+1}\right]$. Compared with the original model (9), the continuous-time system equations are transcribed into a series of algebraic equations. This static version can then be solved by standard optimization techniques [24].

\section{B. Initial Parking Maneuver Planner}

Following the establishment of discretized model, it is desired to find an efficient optimization algorithm. In this paper, a two-stage optimization structure is used to search the optimal solution of Eq.(10). The motivation for the use of this design philosophy relies on its enhanced convergence and computational ability. Traditional optimizers tend to be sensitive with the user-provided initial guess value and they are likely to get stuck at an infeasible point or local optimal point. This issue becomes more severe when the number of decision variable increases. To effectively deal with this issue, an initial parking movement generator is designed. The method used in this stage is an adaptive gradient PSO (AGPSO) method. For completeness, a brief description of this approach is stated below.

PSO is a simple swarm-based intelligent algorithm. Each particle among the swarm has a position as well as a velocity vector. That is,

$$
\begin{aligned}
u_{j}(G) & =\left[u_{j, 1}(G), \ldots, u_{j, D}(G)\right] \\
v_{j}(G) & =\left[v_{j, 1}(G), \ldots, v_{j, D}(G)\right]
\end{aligned}
$$

in which $j=1,2, \ldots, N_{j} ; N_{j}$ stands for the size of the swarm. $D$ denotes the dimensionality index of the searching space. In this way, each particle located at position $u_{j}$ can be treated as a candidate solution. During the evolution process, we can denote the best position of the $j$ th particle as $p_{j}(G)=\left[p_{j, 1}, \ldots, p_{j, D}\right]$, whereas the best position among the swarm can be recorded as $g(G)=\left[g_{1}(G), \ldots, g_{D}(G)\right]$. Subsequently, the new velocity vector is updated according to the definitions of $g$ and $p$, which can be written as:

$$
\begin{aligned}
v_{j, d}(G+1)= & w v_{j, d}(G)+r_{1} c_{1}\left(p_{j, d}(G)-u_{j, d}(G)\right) \\
& r_{2} c_{2}\left(g_{d}(G)-u_{j, d}(G)\right)
\end{aligned}
$$

In Eq.(12), several parameters are introduced. For example, $w$ is the inertia weight parameter and is usually assigned as a constant. $c_{1}$ and $c_{2}$ are two acceleration parameters corresponding to the cognitive component $\left(p_{j, d}(G)-u_{j, d}(G)\right)$ and the social component $\left(g_{d}(G)-u_{j, d}(G)\right)$, respectively. $r_{1}$ and $r_{2}$, on the other hand, are two random parameters defined on $[0,1]$. Based on Eq.(11) and Eq.(12), the new position of the $j$ th particle can be computed via:

$$
u_{j, d}(G+1)=u_{j, d}(G)+v_{j, d}(G+1)
$$

To evaluate the quality of the particle, the fitness function should be introduced. For general unconstrained problems, the fitness value can simply be set as the objective value. However, for the constrained parking maneuver planning problem, the constraint violation value should also be included in the fitness function so as to reflect the solution feasibility. To do this, an effective strategy is to calculate the total violation degree of the particle $\operatorname{Vol}_{j, d}(G) \in[0,1]$. The way to compute this value can be found in [25] and is omitted for space reasons. Consequently, the augmented fitness value associated with each particle is computed via:

$$
J_{j, d}^{a u g}(G)= \begin{cases}J_{j, d}(G), & \text { if } \operatorname{Vol}_{j, d}(G)=0 \\ J^{*}(G)+J^{*}(G) \operatorname{Vol}_{j, d}(G), & \text { if } \operatorname{Vol}_{j, d}(G)>0 .\end{cases}
$$

where $J^{*}(G)$ is the worst objective value among the $G$ th iteration.

It is well known that PSO method has a strong global exploration ability. In order to further enhance its local exploitation ability, a local gradient operation is embedded in the algorithm framework. Supposing that $V o l$ and $J$ are differentiable in their searching space, the Jacobian vector of $V o l$ and $J$ can be expressed by [26]:

$$
\begin{aligned}
\nabla_{u} J_{j} & =\left[\frac{\partial J_{j}}{\partial u_{j, 1}}, \ldots, \frac{\partial J_{j}}{\partial u_{j, d}}\right] \\
\nabla_{u} V o l_{j} & =\left[\frac{\partial V o l_{j}}{\partial u_{j, 1}}, \ldots, \frac{\partial V l_{j}}{\partial u_{j, d}}\right]
\end{aligned}
$$

Based on the Jacobian vector, a local searching direction that minimizes the objective and constraint violation can be written as $e_{j}=-\left(a_{1} \frac{\nabla_{u} J_{j}}{\left\|\nabla_{u} J_{j}\right\|}+a_{2} \frac{\nabla_{u} \text { Vol }_{j}}{\| \nabla_{u} \text { Vol }_{j} \|}\right)$. It is easy to verify that a decrease with regard to the augmented fitness function can be achieved by moving the current position along $e$. Specifically, if one calculates the inner product of $\left\langle e_{j},-\left(\nabla J_{j} /\left\|\nabla J_{j}\right\|\right)\right\rangle$ or $\left\langle e_{j},-\left(\nabla V \operatorname{ol}_{j} /\left\|\nabla V \mathrm{ol}_{j}\right\|\right)\right\rangle$, the result will be negative [26]. It is worth noting that $a_{1}$ and $a_{2}$ are two positive parameters. Since the primary task for the initial parking movement generator is to produce a feasible and nearoptimal reference, these two parameters should be adjusted in an adaptive way. This is achieved by setting $a_{1}=N_{f} / N_{j}$ and $a_{2}=1-a_{1}$. Here, $N_{f}$ refers to the number of feasible candidates among the current generation. In this way, more priorities can be given to optimizing the objective when there are more feasible candidates among the swarm and vice versa. After calculating the descent direction $e_{j}$, the current solution is updated by

$$
\bar{u}_{j}(G)=u_{j}(G)+s_{j} e_{j}
$$

where $s$ stands for the step length along $e_{j}$.

Remark 2. The evolution process of the constructed AGPSO algorithm will continue until the number of generation reaches the limit. Since the goal of the initial movement generator is only to produce a qualified guess for the second stage optimization process, the maximum value of $G$ is limited to $G^{\max }=30$ for the sake of computational burden. 
Remark 3. One advantage of using the AGPSO algorithm in the first stage is that it requires no physical and theoretical knowledge of the problem. Moreover, the robustness and convergence with respect to the particle position and velocity can be guaranteed by selecting the control parameters $w, c_{1}$ and $c_{2}$ properly.

\section{Optimization Strategy in the Second Stage}

The optimization strategy applied in the second stage is traditional gradient method (e.g. sequential quadratic programming or IPM). It was investigated in [25] that if a gradientbased technique starts its Newton iteration from an initial point which is near to the optimal solution, the algorithm can successfully converge to desired point with less iteration and computational time. Therefore, the initial parking maneuver trajectory obtained from the first stage is provided to the second stage gradient-based algorithm as a "warm start". At this point, most of constraints are less likely to become active and the search direction is less restricted.

\section{Implementation consideration of the Two-Stage Opti- mization}

In order to better illustrate the proposed parking maneuver optimization framework, the overall algorithm implementation flowchart is depicted in Fig.2, while the twostage optimization process is extracted and summarised in the pseudocode (see e.g., Algorithm 1).

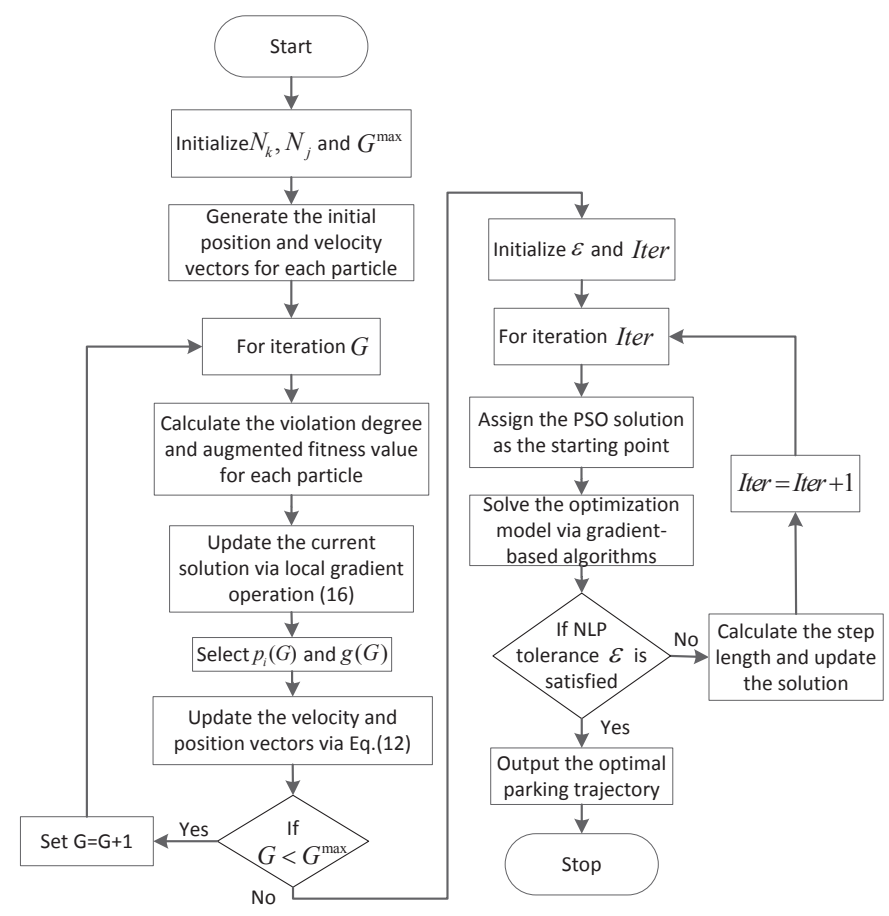

Fig. 2: Overall algorithm implementation flowchart

The two-stage optimization process is implemented over sequential calls to several function files carrying out the particle initialization, computing the local gradient direction, performing the Newton iteration, and calculating the step length. A number of function files are created for different

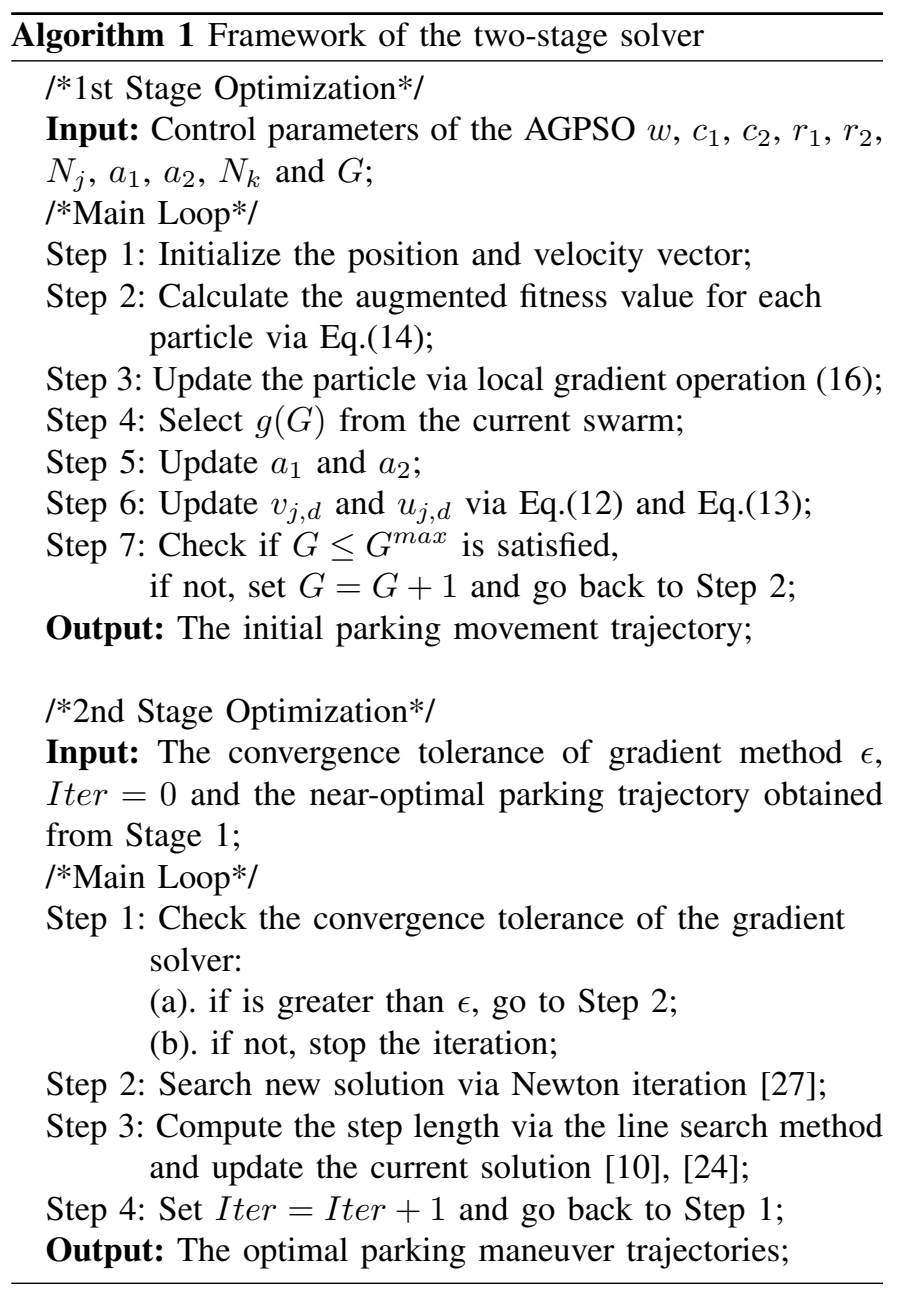

components of the algorithm. For instance, in the first optimization stage, functions are defined to produce:

1) The temporal nodes and discretization coefficients.

2) The initial position and velocity vectors of all the particles.

3) The total violation degree of each particle.

4) The augmented fitness value, $p_{j}(G)$ and $g(G)$.

5) The descent direction $e_{j}$ and the locally updated solution.

Besides, several function files are defined in the second optimization stage so as to calculate:

1) The first and second-order derivatives of the objective function.

2) The derivative of the parking movement path constraints.

3) The step length regulated by the Goldstein condition [24].

\section{SIMULATION STUDY}

In this section, the simulation results of applying the designed two-stage optimization framework to the autonomous ground vehicle parking trajectory planning problem constructed in Sec II are shown. The assignment of vehicle-dependent and mission-dependent parameters is displayed in Table I, whereas control parameters for the proposed optimization scheme are tabulated in Table II.

In order to validate the performance of the proposed design, several mission cases are established and tested. The 
TABLE I: Vehicle/mission dependent parameters

\begin{tabular}{lclc}
\hline \hline Parameters & Values & Variables & Ranges \\
\hline$l_{S L}, \mathrm{~m}$ & 5 & $p_{x}, \mathrm{~m}$ & {$[-10,15]$} \\
$l_{S W}, \mathrm{~m}$ & 2 & $p_{y}, \mathrm{~m}$ & {$[-2,3.5]$} \\
$C L, \mathrm{~m}$ & 3.5 & $v, \mathrm{~m} / \mathrm{s}$ & {$[-2,2]$} \\
$n, \mathrm{~m}$ & 0.8 & $a, \mathrm{~m} / s^{2}$ & {$[-0.75,0.75]$} \\
$l, \mathrm{~m}$ & 2.5 & $\theta, \mathrm{deg}$ & {$\left[-180^{\circ}, 180^{\circ}\right]$} \\
$m, \mathrm{~m}$ & 0.7 & $\phi, \mathrm{deg}$ & {$\left[-33^{\circ}, 33^{\circ}\right]$} \\
$2 b, \mathrm{~m}$ & 1.771 & $t, \mathrm{~s}$ & {$[0,50]$} \\
\hline \hline
\end{tabular}

TABLE II: Control parameters of the algorithm

\begin{tabular}{lclc}
\hline \hline Parameters & Values/ranges & Parameters & Values/ranges \\
\hline$w$ & $\left(1+r_{1}\right) / 2$ & $a_{1}$ & {$[0,1]$} \\
$c_{1}$ & 1.49445 & $a_{2}$ & {$[0,1]$} \\
$c_{2}$ & 1.49445 & $N_{k}$ & 50 \\
$r_{1}$ & {$[0,1]$} & $G^{\max }$ & 30 \\
$r_{2}$ & {$[0,1]$} & $\epsilon$ & $10^{-6}$ \\
$N_{j}$ & 100 & Iter $^{\max }$ & 5000 \\
\hline \hline
\end{tabular}

initial conditions, along with the collision-free constraint setting for different case studies, are summarised in Table III. The positional information for different obstacles $O^{p}, p=1, \ldots, 6$ is given below:
$O^{1}\left\{\begin{array}{l}A_{x}=6.01, A_{y}=2.61 \\ B_{x}=9.95, B_{y}=3.30 \\ C_{x}=9.64, C_{y}=5.05 \\ D_{x}=5.70, D_{y}=4.35\end{array}\right.$
$O^{2}\left\{\begin{array}{l}A_{x}=8.03, A_{y}=0.66 \\ B_{x}=11.97, B_{y}=-0.03 \\ C_{x}=11.66, C_{y}=-1.78 \\ D_{x}=7.72, D_{y}=-1.08\end{array}\right.$
$O^{3}\left\{\begin{array}{l}A_{x}=0.27, A_{y}=-0.92 \\ B_{x}=-3.59, B_{y}=-1.96 \\ C_{x}=-4.05, C_{y}=-0.24 \\ D_{x}=-0.19, D_{y}=0.79\end{array}\right.$
$O^{4}\left\{\begin{array}{l}A_{x}=-1.35, A_{y}=2.53 \\ B_{x}=2.21, B_{y}=0.71 \\ C_{x}=3.02, C_{y}=2.29\end{array}\right.$
$O^{5}\left\{\begin{array}{l}A_{x}=5.25, A_{y}=0.50 \\ B_{x}=9.18, B_{y}=1.26 \\ C_{x}=9.51, C_{y}=0.48 \\ D_{x}=5.59, D_{y}=-1.24\end{array}\right.$
$\left\{\begin{array}{l}C_{x}=3.02, C_{y}=2.29 \\ D_{x}=-0.55, D_{y}=4.11\end{array}\right.$
$O^{6}\left\{\begin{array}{l}A_{x}=0.52, A_{y}=-1.25 \\ B_{x}=-3.43, B_{y}=-1.91 \\ C_{x}=-3.72, C_{y}=-0.16 \\ D_{x}=0.22, D_{y}=0.50\end{array}\right.$

TABLE III: Automatic parking mission cases

\begin{tabular}{clc}
\hline \hline Case No. & Initial conditions & Obstacle position $O^{i}$ \\
\hline 1 & $\left\{\begin{array}{l}p_{x}(0)=10.70 \\
p_{y}(0)=1.5 \\
\theta(0)=0\end{array}\right.$ & No obstacle \\
\hline 2 & $\left\{\begin{array}{l}p_{x}(0)=10.70 \\
p_{y}(0)=1.5 \\
\theta(0)=0\end{array}\right.$ & Obstacles $1 \& 2$ \\
\hline 3 & $\left\{\begin{array}{l}p_{x}(0)=10.70 \\
p_{y}(0)=1.5 \\
\theta(0)=0\end{array}\right.$ & Obstacles $1 \& 3$ \\
\hline 5 & $\left\{\begin{array}{l}p_{x}(0)=10.70 \\
p_{y}(0)=1.5 \\
\theta(0)=0\end{array}\right.$ & Obstacles $1 \& 2 \& 3$ \\
\hline 5 & $\left\{\begin{array}{l}p_{x}(0)=9.70 \\
p_{y}(0)=2.40 \\
\theta(0)=-5\end{array}\right.$ & Obstacles $4 \& 5$ \\
\hline $\begin{array}{l}p_{x}(0)=9.70 \\
p_{y}(0)=2.4 \\
\theta(0)=-5\end{array}$ & Obstacles $4 \& 5 \& 6$ \\
\hline \hline
\end{tabular}

The state boundary values at the terminal time $t_{f}$ should satisfy $x_{f}=\left[v_{f}, a_{f}\right]=[0,0]$. The control constraints assigned to the jerk and curvature derivative are given by jerk $(t) \in$ $[-0.5,0.5]$ and $\dot{k}(t) \in[-0.6,0.6]$, respectively. All the simulation results were generated applying Matlab 2016b under Windows 10 and Intel (R) Core(TM) i7-4790 CPU, with 12.00 GB RAM.

\section{A. Parameter analysis}

In this subsection, the impact of different control parameters on the computational time and convergence ability is firstly studied. This analysis includes: 1). the influence of the number of temporal node $N_{k}$; and 2). the impact of the convergence tolerance value $\epsilon$.

To assess the convergence speed of the AGPSO optimization with respect to $N_{k}$, the goal attainment value is used as the primary indicator. Take mission case 2 as an example, the goal attainment value $\mu_{t_{f}} \in[0,1]$ is computed by $\mu_{t_{f}}=1-\frac{J^{\text {aug }}-t_{f}^{*}}{t_{f}^{\text {max }}-t_{f}^{*}}$, where $t_{f}^{*}$ and $t_{f}^{\max }$ are set to 15 and 20 , respectively. By specifying $N_{k}=(50,75,100,150)$, the results are depicted in Fig.3.

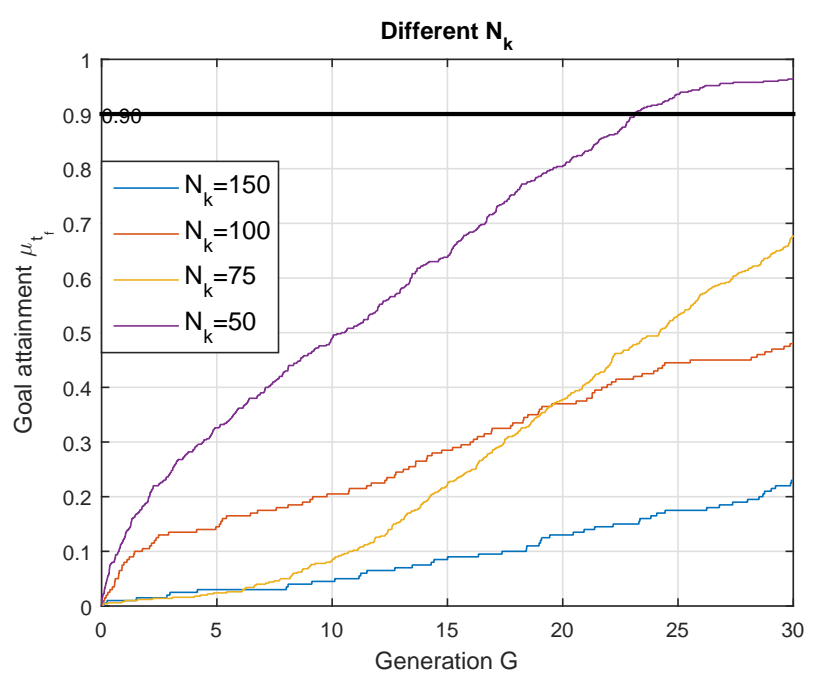

Fig. 3: Convergence results with respect to $N_{k}$

Fig. 3 presents the results on the goal attainment value versus the number of generation $G$ plane. Although using a large $N_{k}$ can improve the approximation accuracy of the continuous-time model, the number of decision variables will be increased. As a result, the convergence speed of the AGPSO is decreased significantly. Since the aim of the first stage optimization is only to produce a near-optimal parking movement to warm start the second stage optimization, a relatively small $N_{k}$ value (e.g., $N_{k}=50$ ) is used.

Furthermore, it is found that the second stage optimization process tends to be sensitive with respect to the convergence tolerance value $\epsilon$. Using case 1 as an instance, a sensitivity analysis was performed by specifying different values of $\epsilon$ and the results are displayed in Table IV. According

TABLE IV: Sensitivity analysis with respect to $\epsilon$

\begin{tabular}{l|c|c|c|c|c|}
\hline \hline Values for $\epsilon$ & $1 \mathrm{e}-4$ & $1 \mathrm{e}-5$ & $1 \mathrm{e}-6$ & $1 \mathrm{e}-7$ & $1 \mathrm{e}-8$ \\
\hline$t_{f}(\mathrm{~s})$ & 15.072 & 15.045 & 14.140 & 14.128 & 14.122 \\
$t_{p}(\mathrm{~s})$ & 44.384 & 44.775 & 45.324 & 66.541 & 82.337 \\
\hline \hline
\end{tabular}

to Table IV, a better solution can be obtained by using a smaller index of accuracy $\epsilon$. However, the computational time $t_{p}$ is monotonically increasing as $\epsilon$ becomes tighter. To balance the solution optimality and the computational burden, $\epsilon$ is set to $10^{-6}$ for the test trials. 


\section{B. Optimal Parking Maneuver Trajectories}

The optimal parking maneuver trajectory for different mission cases are partly displayed and the effectiveness of the obtained results is analyzed in this subsection. More precisely, the maneuver profiles for cases 3-6 are plotted in Fig.4, whereas the corresponding optimized state and control profiles are depicted in Figs.5-9. Different from most previous works [14], [28], wherein regularly parked obstacles were considered, we are interested in finding optimal parking trajectories with the consideration of irregularly placed obstacles. Moreover, in case 4 and case 6 , the target parking area is occupied partly by other vehicles. As can be seen from Figs.4-9, the proposed two-stage optimization framework is able to optimize the parking maneuver without violating the vehicle path, terminal and collision-free constraints. In terms of the solution optimality, this can be partly reflected by the calculated control evolution profiles (especially the jerk trajectory). Since the jerk variable does not appear in the path constraint and it appears in the dynamics linearly, we can expect the jerk has a bangsingular-bang behaviour for any $t \in\left[0, t_{f}\right]$. This conclusion can also be analogized according to the Proposition 3 stated in [10].
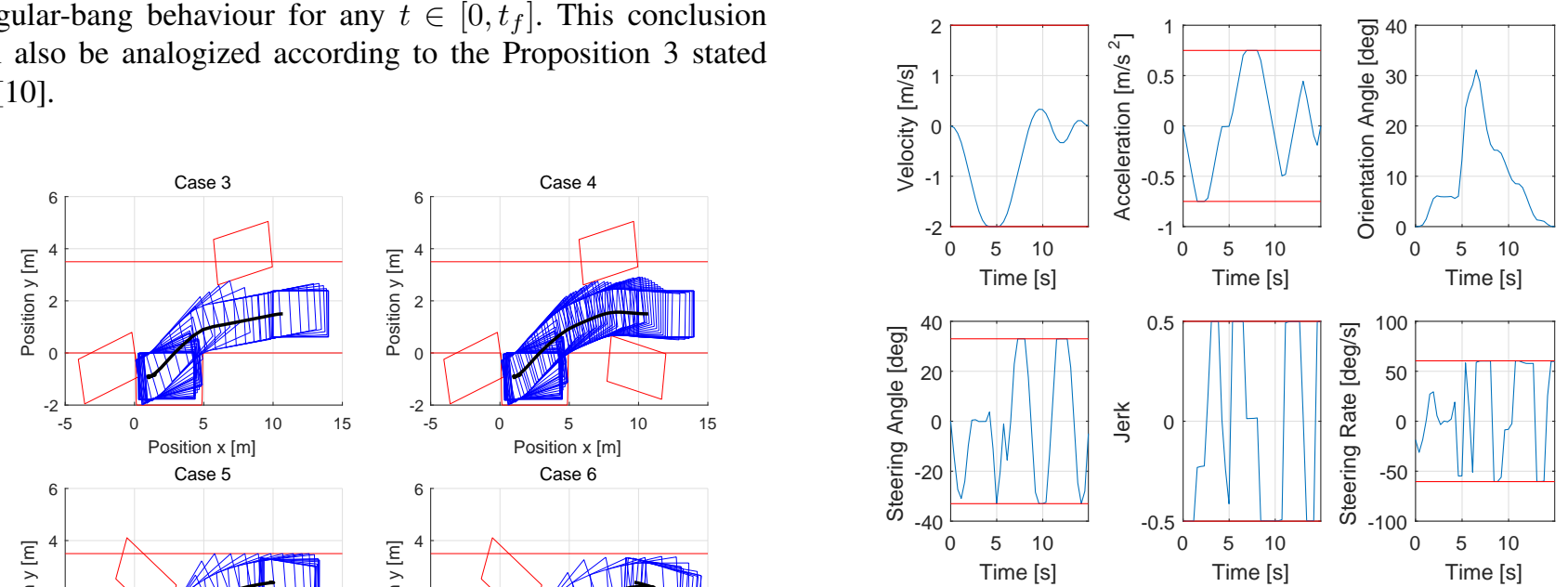

limited rooms to adjust its attitude. As a result, to meet the specific final boundary conditions, more number of maneuver are required at the expense of objective value (e.g. final time $\left.t_{f}\right)$.

\section{Comparison Against Other Techniques}

This subsection presents a comparative study in terms of the optimal parking movement achieved by performing the proposed two-stage strategy and other optimization-based trajectory planning techniques reported in the literature. For instance, an IPM-based direct transcription method outlined in [15] and an artificial bee colony-based (ABC) intelligent
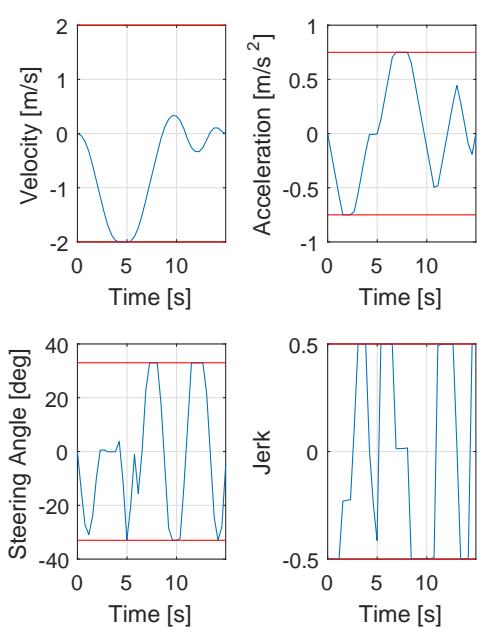

Fig. 6: Optimal trajectories for Case 3
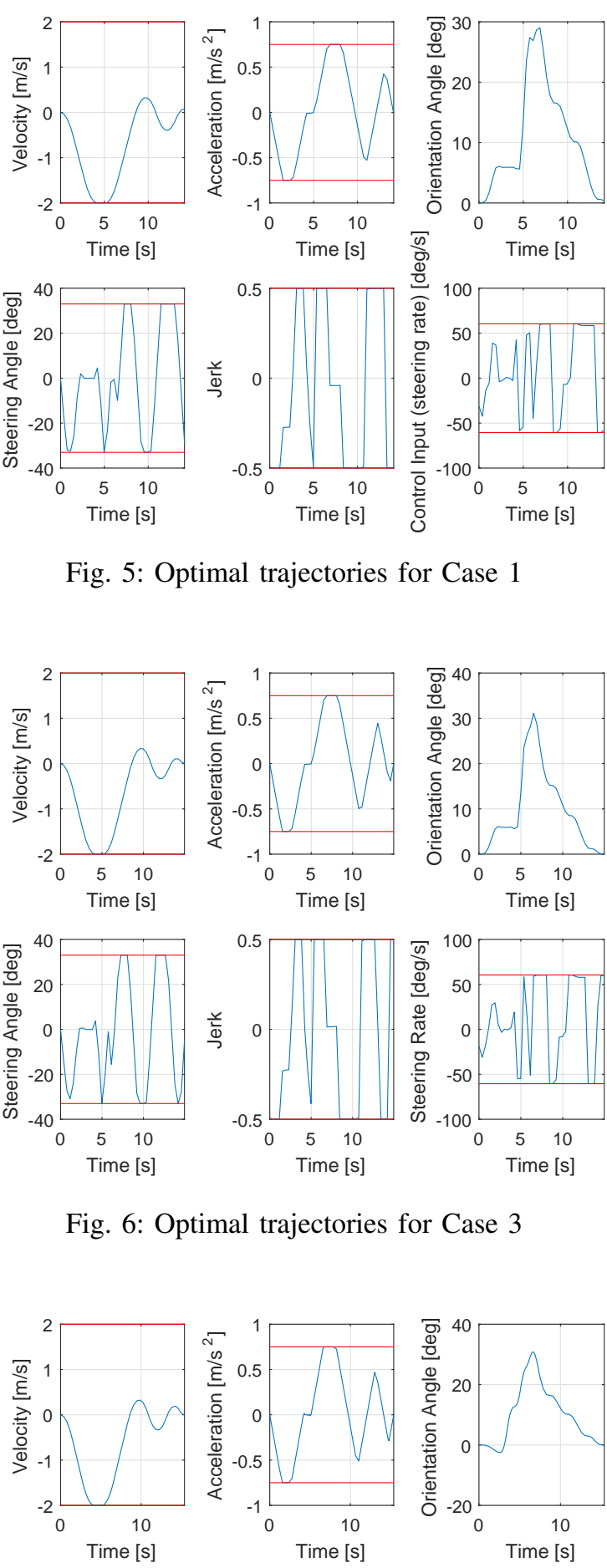

Fig. 5: Optimal trajectories for Case 1
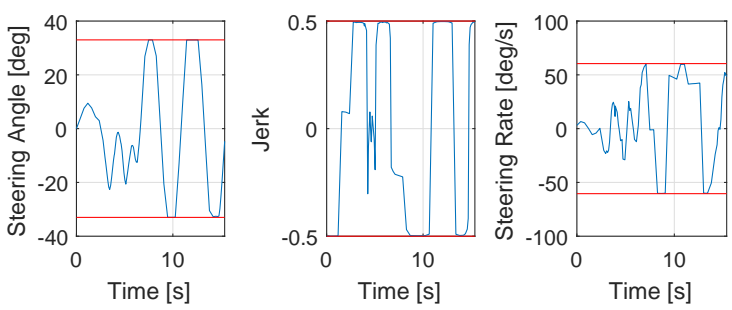

Fig. 7: Optimal trajectories for Case 4 

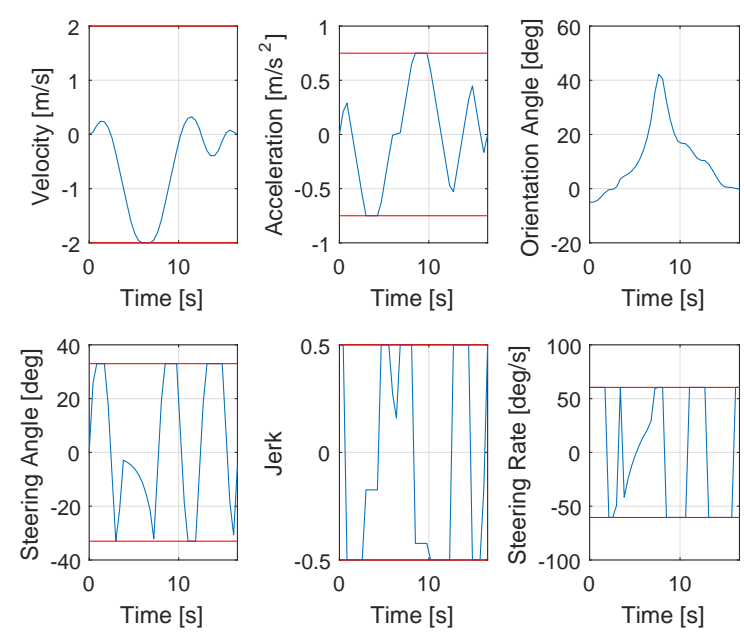

Fig. 8: Optimal trajectories for Case 5
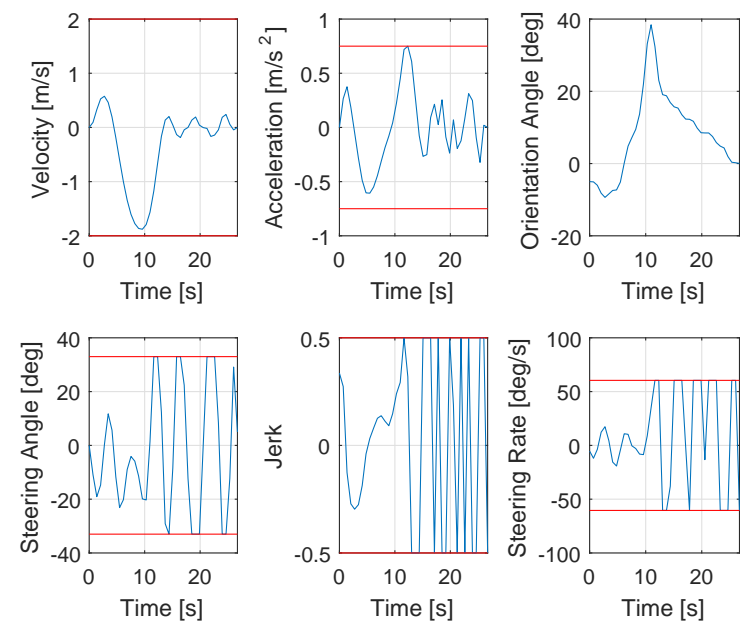

Fig. 9: Optimal trajectories for Case 6

optimization method studied in [29]. For the purpose of comparison, the default setting suggested in the original paper is used. Detailed optimization results including the objective and process time $t_{p}$ for different parking scenarios are summarised in Table V.

TABLE V: Computational results for different methods

\begin{tabular}{c|cc|cc|cc}
\hline Case & \multicolumn{2}{|c|}{ IPM-based } & \multicolumn{2}{c|}{ ABC-based } & \multicolumn{2}{c}{ Proposed } \\
\cline { 2 - 7 } No. & $t_{f}(\mathrm{~s})$ & $t_{p}(\mathrm{~s})$ & $t_{f}(\mathrm{~s})$ & $t_{p}(\mathrm{~s})$ & $t_{f}(\mathrm{~s})$ & $t_{p}(\mathrm{~s})$ \\
\hline 1 & 14.140 & 27.996 & 15.091 & 157.263 & 14.140 & 45.324 \\
\hline 2 & 14.943 & 179.311 & 15.224 & 163.408 & 14.929 & 68.232 \\
\hline 3 & 14.982 & 222.891 & 15.576 & 161.367 & 14.955 & 76.734 \\
\hline 4 & 15.558 & 264.382 & 15.971 & 159.192 & 15.374 & 88.380 \\
\hline 5 & 16.707 & 211.600 & 17.053 & 147.135 & 16.569 & 75.121 \\
\hline 6 & 27.147 & 289.523 & 28.506 & 156.381 & 26.723 & 147.192 \\
\hline
\end{tabular}

It is important to note that in this study, we are interested in finding an optimization strategy such that the computational time $t_{p}$ can be decreased yet the solution optimality can be maximally preserved. Hence, compared with the $t_{f}$ results, $t_{p}$ is rather important and should be given more attentions when assessing the performance of different algorithms. From the results presented in Table $\mathrm{V}$, it can be seen that the optimal objective results $t_{f}$ calculated using the proposed method and single-stage IPM-based approach investigated in [15] are comparable. For the normal case (e.g. case 1), the IPM-based direct method can generate the parking trajectory with the smallest computational cost. However, when more irregular collision-free constraints are considered in the optimization model, it becomes difficult for a single-stage optimization structure to converge. Alternatively, the two-stage optimization framework tends to perform better in terms of achieving enhanced convergence speed (small $t_{p}$ values) and objective values. This further confirms the superiority of using the investigated scheme to compute the optimal parking maneuver.

Apart from the objective value, attentions should also be given to the passenger's comfort of the obtained solutions. To assess the comfort of passengers, certain evaluation metrics are desired. It is worth noting that one important factor that could have significant influences with respect to the passenger's comfort is the path smoothness. By applying the information of the jerk and $\dot{k}$, a comfort indicator and a path smoothness indicator are defined. For example, the peak jerk value $\operatorname{Ind}_{1}=\max (|j e r k|)$ and $I n d_{2}=\int_{0}^{t_{f}} \dot{k} d t$, respectively. The comparative results for different parking scenarios are summarised and tabulated in Table VI.

TABLE VI: Comparative results for different methods

\begin{tabular}{|c|c|c|c|c|c|c|}
\hline Case & \multicolumn{2}{|c|}{ IPM-based } & \multicolumn{2}{|c|}{ ABC-based } & \multicolumn{2}{|c|}{ Proposed } \\
\hline No. & $\operatorname{Ind}_{1}$ & $\operatorname{Ind} d_{2}$ & $I n d_{1}$ & $\operatorname{Ind}_{2}$ & Ind & $\operatorname{Ind}_{2}$ \\
\hline 1 & 0.50 & 1.0720 & 0.50 & 1.2175 & 0.50 & 1.0720 \\
\hline 2 & 0.50 & 0.3063 & 0.50 & 0.5384 & 0.50 & 0.3061 \\
\hline 3 & 0.50 & 1.0024 & 0.50 & 1.4783 & 0.50 & 1.0049 \\
\hline 4 & 0.50 & 0.6831 & 0.50 & 0.7347 & 0.50 & 0.6775 \\
\hline 5 & 0.50 & 0.0805 & 0.50 & 0.1177 & 0.50 & 0.0798 \\
\hline 6 & 0.50 & 2.2058 & 0.48 & 2.8562 & 0.50 & 2.0141 \\
\hline
\end{tabular}

As can be observed from Table VI, all the algorithms can produce the parking trajectory without violating the jerk path constraint. The proposed method can produce smoother results than its counterparts for most test cases. Interestingly, a relatively uneven performance can be found in the ABC-based results. This is because it applies the random initialization process and stochastic evolutionary strategies. Hence, it tends to contain more fluctuations with respect to the control variables [24].

Remark 4. From Table V, the computational cost results obtained via a single gradient optimizer might experience a significant variance between different cases. This is mainly caused by the collision-free constraints. One obstacle will result in eight path constraints and the increasing number of constraints entailed in the optimization model will tighter the searching space of the optimization process which in turn slow down the convergence speed.

\section{Convergence and Robustness Analysis}

Another attempt is carried out so as to analyze the convergence ability and robustness of the proposed twostage optimization structure. A dispersion experiment was performed for the parking case 5 with 1000 Monto-Carlo trials. The initial conditions of the vehicle are perturbed and the random initialization data are summarised in Table VII. 
TABLE VII: Dispersions of initial conditions

\begin{tabular}{lcc}
\hline \hline Initial states & Distribution & $3-\sigma$ range \\
\hline$p_{x}(0), \mathrm{m}$ & Uniform & $\pm 5 \%$ \\
$p_{x}(0), \mathrm{m}$ & Uniform & $\pm 5 \%$ \\
$v(0), \mathrm{m} / \mathrm{s}$ & Zero-mean Gaussian & 0.25 \\
$a(0), \mathrm{m} / \mathrm{s}^{2}$ & Zero-mean Gaussian & 0.25 \\
$\theta(0), \mathrm{deg}$ & Uniform & $\pm 5 \%$ \\
$\phi(0), \mathrm{deg}$ & Uniform & $\pm 5 \%$ \\
\hline \hline
\end{tabular}

Comparative study was made to analyze the performance of different gradient optimization strategies, such as the single IPM-based and single SQP-based and the two-stage optimization framework. It is important to mention that in this test, the maximum number of Newton iteration Iter ${ }^{\max }$ is limited to 500. The convergence results of the dispersion experiment are established in Table VIII.

TABLE VIII: Convergence results for different strategies

\begin{tabular}{l|c|c|c|c}
\hline \hline Different methods & $N_{s}$ & $N_{i n f}$ & $N_{m}$ & $\begin{array}{l}\text { Successful } \\
\text { rate (\%) }\end{array}$ \\
\hline $\begin{array}{l}\text { Single optimization } \\
\text { (Pure SQP) }\end{array}$ & 183 & 116 & 701 & 18.3 \\
\hline $\begin{array}{l}\text { Single optimization } \\
\text { (Pure IPM) }\end{array}$ & 210 & 78 & 712 & 21.0 \\
\hline $\begin{array}{l}\text { Two-stage optimization } \\
\text { (AGPSO+SQP) }\end{array}$ & 719 & 28 & 253 & 71.9 \\
\hline $\begin{array}{l}\text { Two-stage optimization } \\
\text { (AGPSO+IPM) }\end{array}$ & 733 & 19 & 248 & 73.3 \\
\hline \hline
\end{tabular}

As can be observed from Table VIII, three performance indicators are included. That is, the times of successful solution found $N_{s}$, the times of infeasible solution detected $N_{\text {inf }}$, and the times of maximum iteration exceeded $N_{m}$. It is obvious that compared with SQP optimization method, the IPM tends to have better solution-finding ability for the problem investigated in this study. This can be reflected by the fact that $N_{s}$ achieved using IPM is generally larger than that of SQP method. Besides, the two-stage approach is able to achieve a higher successful rate with respect to finding optimal solutions compared with the single optimization structure. Therefore, it can be concluded that the implementation of stage 1 (initial parking movement generator) can have positive influences for improving the computational time and convergence ability of the parking optimization process.

\section{Concluding Remarks and Future works}

In this investigation, a hybrid optimization structure was constructed and applied to address the autonomous vehicle parking motion planning problem. In order to effectively handle the sensitivity issue, an initial parking movement generator based on an adaptive gradient particle swarm optimization algorithm was designed. In this way, the stage-two gradient-based solver can start the solution-finding iteration at a near-optimal point, thereby improving the computational efficiency as well as the convergence ability. This conclusion was verified by carrying out a number of case studies and comparative simulations. Moreover, dispersion experiments were also performed and the results revealed the proposed two-stage strategy can have a more robust performance than traditional single-stage optimization structures.
Our follow-up work will focus on enhancing the stability of the proposed two-stage optimization algorithm such that it can be applied in dealing with more complicated vehicle models and automatic driving mission scenarios. Furthermore, it would be worthwhile to take into account some potential model errors and environmental uncertainties during the optimization process. Moreover, an integrated parking trajectory planning and tracking control system will also be designed by applying multi-layer optimization-based techniques.

\section{REFERENCES}

[1] G. Demesure, M. Defoort, A. Bekrar, D. Trentesaux, and M. Djemai, "Decentralized motion planning and scheduling of agvs in an fms," IEEE Transactions on Industrial Informatics, vol. 14, no. 4, pp. 1744-1752, 2018.

[2] H. Guo, C. Shen, H. Zhang, H. Chen, and R. Jia, "Simultaneous trajectory planning and tracking using an mpc method for cyber-physical systems: A case study of obstacle avoidance for an intelligent vehicle," IEEE Transactions on Industrial Informatics, pp. 1-1, 2018.

[3] Y. Wang, S. Wang, and M. Tan, "Path generation of autonomous approach to a moving ship for unmanned vehicles," IEEE Transactions on Industrial Electronics, vol. 62, no. 9, pp. 5619-5629, 2015.

[4] A. Rucco, G. Notarstefano, and J. Hauser, "An efficient minimumtime trajectory generation strategy for two-track car vehicles," IEEE Transactions on Control Systems Technology, vol. 23, no. 4, pp. 15051519, 2015.

[5] B. Tian, W. Fan, R. Su, and Q. Zong, "Real-time trajectory and attitude coordination control for reusable launch vehicle in reentry phase," IEEE Transactions on Industrial Electronics, vol. 62, no. 3, pp. 1639-1650, 2015.

[6] G. Xie, H. Gao, L. Qian, B. Huang, K. Li, and J. Wang, "Vehicle trajectory prediction by integrating physics- and maneuver-based approaches using interactive multiple models," IEEE Transactions on Industrial Electronics, vol. 65, no. 7, pp. 5999-6008, 2018.

[7] M. Liu, "Robotic online path planning on point cloud," IEEE Transactions on Cybernetics, vol. 46, no. 5, pp. 1217-1228, 2016.

[8] Y. Kim and B. K. Kim, "Time-optimal trajectory planning based on dynamics for differential-wheeled mobile robots with a geometric corridor," IEEE Transactions on Industrial Electronics, vol. 64, no. 7, pp. $5502-5512,2017$.

[9] X. Chu, Q. Hu, and J. Zhang, "Path planning and collision avoidance for a multi-arm space maneuverable robot," IEEE Transactions on Aerospace and Electronic Systems, vol. 54, no. 1, pp. 217-232, 2018.

[10] R. Chai, A. Savvaris, A. Tsourdos, S. Chai, and Y. Xia, "Trajectory optimization of space maneuver vehicle using a hybrid optimal control solver," IEEE Transactions on Cybernetics, vol. PP, no. 99, pp. 1-14, 2017.

[11] R. Chai, A. Savvaris, and A. Tsourdos, "Fuzzy physical programming for space manoeuvre vehicles trajectory optimization based on hp-adaptive pseudospectral method," Acta Astronautica, vol. 123, pp. 62-70, 2016.

[12] X. Yin and Q. Chen, "Trajectory generation with spatio-temporal templates learned from demonstrations," IEEE Transactions on Industrial Electronics, vol. 64, no. 4, pp. 3442-3451, 2017.

[13] B. Muller, J. Deutscher, and S. Grodde, "Continuous curvature trajectory design and feedforward control for parking a car," IEEE Transactions on Control Systems Technology, vol. 15, no. 3, pp. 541-553, 2007.

[14] C.-K. Lee, C.-L. Lin, and B.-M. Shiu, "Autonomous vehicle parking using hybrid artificial intelligent approach," Journal of Intelligent and Robotic Systems, vol. 56, no. 3, pp. 319-343, 2009.

[15] B. Li and Z. Shao, "Simultaneous dynamic optimization: A trajectory planning method for nonholonomic car-like robots," Advances in Engineering Software, vol. 87, pp. 30-42, 2015.

[16] V. Roberge, M. Tarbouchi, and G. Labonte, "Comparison of parallel genetic algorithm and particle swarm optimization for real-time uav path planning," IEEE Transactions on Industrial Informatics, vol. 9, no. 1, pp. 132-141, 2013.

[17] J. J. Kim and J. J. Lee, "Trajectory optimization with particle swarm optimization for manipulator motion planning," IEEE Transactions on Industrial Informatics, vol. 11, no. 3, pp. 620-631, 2015.

[18] B. Li, K. Wang, and Z. Shao, "Time-optimal maneuver planning in automatic parallel parking using a simultaneous dynamic optimization approach," IEEE Transactions on Intelligent Transportation Systems, vol. 17, no. 11, pp. 3263-3274, 2016. 
[19] A. Reiter, A. Muller, and H. Gattringer, "On higher order inverse kinematics methods in time-optimal trajectory planning for kinematically redundant manipulators," IEEE Transactions on Industrial Informatics, vol. 14, no. 4, pp. 1681-1690, 2018.

[20] A. J. Hausler, A. Saccon, A. P. Aguiar, J. Hauser, and A. M. Pascoal, "Energy-optimal motion planning for multiple robotic vehicles with collision avoidance," IEEE Transactions on Control Systems Technology, vol. 24, no. 3, pp. 867-883, 2016.

[21] F. You, R. Zhang, G. Lie, H. Wang, H. Wen, and J. Xu, "Trajectory planning and tracking control for autonomous lane change maneuver based on the cooperative vehicle infrastructure system," Expert Systems with Applications, vol. 42, no. 14, pp. 5932-5946, 2015.

[22] R. Zhang, Z. He, H. Wang, F. You, and K. Li, "Study on self-tuning tyre friction control for developing main-servo loop integrated chassis control system," IEEE Access, vol. 5, pp. 6649-6660, 2017.

[23] R. Zhang, K. Li, Z. He, H. Wang, and F. You, "Advanced emergency braking control based on a nonlinear model predictive algorithm for intelligent vehicles," Applied Sciences, vol. 7, no. 5, 2017.

[24] R. Chai, A. Savvaris, A. Tsourdos, S. Chai, and Y. Xia, "Improved gradient-based algorithm for solving aeroassisted vehicle trajectory optimization problems," Journal of Guidance, Control, and Dynamics, vol. 40, no. 8, pp. 2093-2101, 2017.

[25] R. Chai, A. Savvaris, and A. Tsourdos, "Violation learning differential evolution-based hp-adaptive pseudospectral method for trajectory optimization of space maneuver vehicle," IEEE Transactions on Aerospace and Electronic Systems, vol. 53, no. 4, pp. 2031-2044, 2017.

[26] R. Chai, A. Savvaris, A. Tsourdos, S. Chai, and Y. Xia, "Unified multiobjective optimization scheme for aeroassisted vehicle trajectory planning," Journal of Guidance, Control, and Dynamics, vol. 41, no. 7, pp. 1521-1530, 2018.

[27] A. Wachter and L. T. Biegler, "On the implementation of an interiorpoint filter line-search algorithm for large-scale nonlinear programming," Mathematical Programming, vol. 106, no. 1, pp. 25-57, 2006.

[28] F. Gomez-Bravo, F. Cuesta, A. Ollero, and A. Viguria, "Continuous curvature path generation based on b-spline curves for parking manoeuvres," Robotics and Autonomous Systems, vol. 56, no. 4, pp. 360-372, 2008.

[29] H. Duan and S. Li, "Artificial bee colony based direct collocation for reentry trajectory optimization of hypersonic vehicle," IEEE Transactions on Aerospace and Electronic Systems, vol. 51, no. 1, pp. 615-626, 2015.

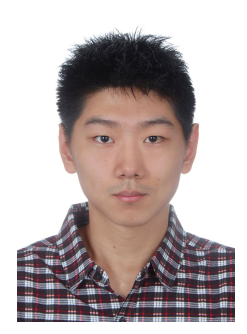

Runqi Chai (SM'15-M'18) was born in Beijing, China, in 1993. He received the Ph.D. degree in Aerospace Engineering at Cranfield University. His research interests include trajectory optimization, guidance and control.

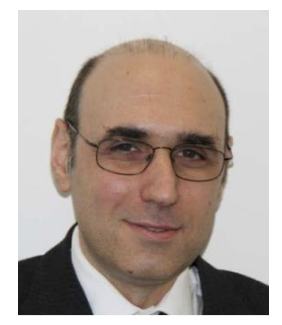

Antonios Tsourdos obtained a Ph.D. on nonlinear robust missile autopilot design and analysis from Cranfield University, in 1999. He is a Professor of Autonomous Systems and Control with Cranfield University. He was appointed Head of the Autonomous Systems Group in 2007, Head of the Centre of Autonomous and Cyber-Physical Systems in 2012 and Director of Research - Aerospace, Transport and Manufacturing in 2015. He leads the research theme on autonomous systems within the School of Aerospace, Transport and Manufacturing at Cranfield University. He has diverse expertise in both unmanned and autonomous vehicles as well as networked systems. He conducts basic and applied research in the fields of guidance, control and navigation for single and multiple unmanned autonomous vehicles as well as research on cyber-physical systems.

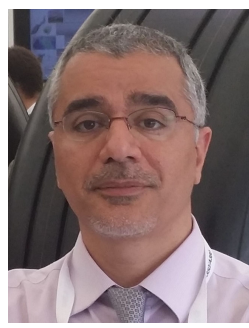

Al Savvaris received the M.Eng. in aerospace systems engineering from the University of Hertfordshire, in 1998, and a Ph.D. on radiowave propagation and system design from University of South Wales, in 2004. He is a Reader in the Centre for Cyber-Physical Systems at Cranfield University. He established the Autonomous Vehicle Dynamics and Control MSc course and the COMAC training programme at Cranfield. His research interests and activities include systems integration, hybrid energy management, communication systems, embedded systems, guidance and control. He was a member of the Autonomous Systems National Technical Committee; EPSRC College review member and reviewer on several international publications including IMechE and IEEE. He has published over 100 peer-reviewed journal and conference papers.

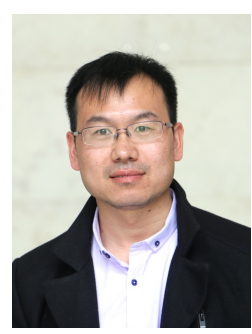

Senchun Chai received his Ph.D. degree and then held a Postdoc fellowship with School of Electronics, University of Glamorgan, UK, in 2007 and 2009, respectively. His current research interests include design of unmanned aerial vehicles (UAV), wireless sensor network control, networked control systems and multiagent control systems.

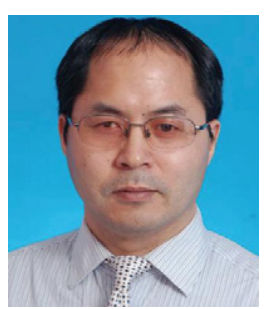

Yuanqing Xia was born in Anhui Province, China, in 1971. He received the B.S. degree from the Department of Mathematics, Chuzhou University, Chuzhou, China, in 1991, the M.S. degree in fundamental mathematics from Anhui University, Wuhu, China, in 1998, and the Ph.D. degree in control theory and control engineering from the Beijing University of Aeronautics and Astronautics, Beijing, China, in 2001. His current research interests are in the fields of networked control systems, robust control and signal processing, active disturbance rejection control and flight control. He has published 8 monographs with Springer and Wiley, and more than 200 papers in journals. He is a Deputy Editor of the Journal of the Beijing Institute of Technology, Associate Editor of Acta Automatica Sinica, Control Theory and Applications, the International Journal of Innovative Computing, Information and Control, and the International Journal of Automation and Computing. 
2018-11-26

Two-stage trajectory optimization for autonomous ground vehicles parking maneuver

Chai, Runqi

IEEE

Runqi Chai, Antonios Tsourdos, Al Savvaris et al., Two-stage trajectory optimization for autonomous ground vehicles parking maneuver. IEEE Transactions on Industrial Informatics, Volume 15, Issue 7, 2019, pp. 3899-3909

https://doi.org/10.1109/TII.2018.2883545

Downloaded from Cranfield Library Services E-Repository 